\title{
CXCR7 activation overrides lung fibrosis
}

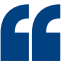 \\ TC14012 attenuated lung fibrosis and Jag 1 induction in mice with various levels of $\beta$-cateinin- induced JAG 1 expression in PCECS}

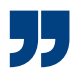

Chronic or repeated lung injury can lead to fibrosis. Now, Ding and colleagues have identified components in the vascular niche that control this process, including chemokine receptor CXCR7. In mice, a CXCR7 agonist promoted alveolar repair and reduced fibrosis after repetitive lung injury.

The microvascular niche contains numerous cells, including pulmonary capillary endothelial cells (PCECs), fibroblasts and macrophages, which communicate with each other through secreted factors. Intervening in these communication pathways is an attractive approach to induce lung regeneration and alleviate fibrosis.

Ding and colleagues sought to investigate the factors involved in switching from the repair-promoting microenvironment that exists following single lung injury to the fibrosis-promoting environment

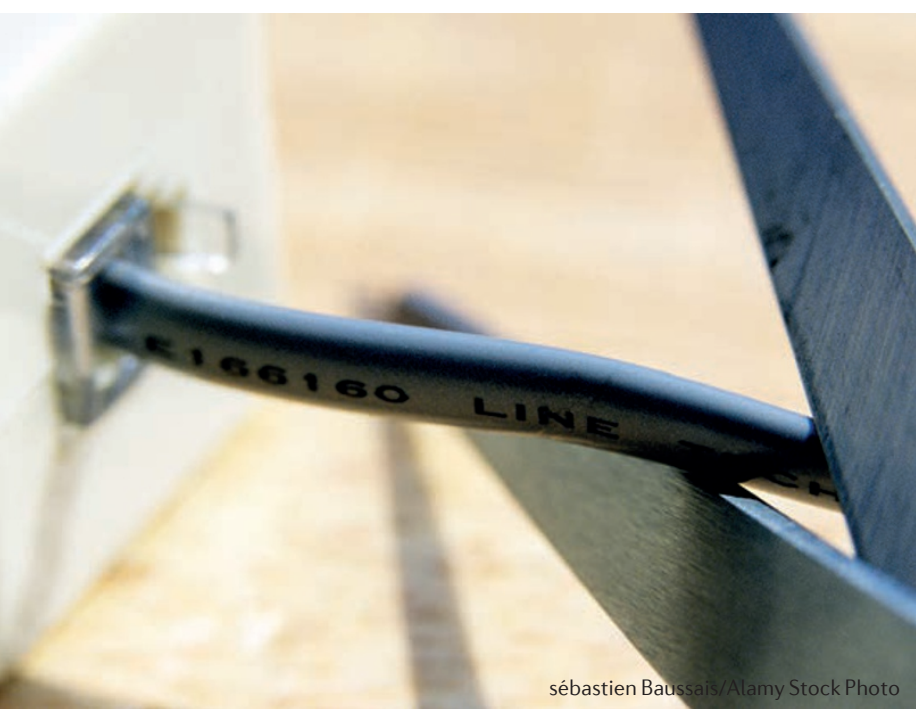

that dominates after multiple injuries. Using a repetitive intratracheal bleomycin injection model in mice (6 injections at 12-day intervals), the authors found that Notch signalling was activated in the fibroblasts of repeatedly damaged lungs.

This activation was associated with increased expression of Jagged 1 (JAG1), a Notch ligand, in PCECs. Indeed, fibrosis of the lung - as measured by levels of smooth muscle actin and collagen I - and Notch signalling in fibroblasts were both attenuated in mice with JAG1-deficient PCECs.

To identify the cause of JAG1 upregulation in PCECs, the authors examined CXCR7, which is expressed in endothelial cells, promotes liver repair and decreases atherosclerosis. They found that levels of this protein were decreased in mice that were repeatedly injected with bleomycin and in patients with pulmonary fibrosis. The CXCR7 ligand, CXCL12, was upregulated in mice that received a single bleomycin injection, suggesting that CXCL12 and its interaction with CXCR7 could promote acute lung repair. Intratracheal infusion with the CXCR7 agonist TC14012 after the third bleomycin injection reduced fibrosis and epithelial damage. Levels of pro-fibrotic JAG1 in PCECs decreased after TC14102 treatment, and this effect of TC14012 was absent after ablation of CXCR7 in endothelial cells, suggesting that CXCR7 controls JAG1 levels in PCECs.

In chronic lung damage, macrophages can home to the site of injury and promote inflammation, which contributes to fibrosis. In their repeated bleomycin-injection model, depletion of macrophages and monocytes at later stage also prevented fibrosis. However, mice in which PCECs expressed a stablized form of $\beta$-catenin, a molecule involved in upregulation of JAG1 expression, still developed fibrosis in the absence of macrophages and monocytes. The authors speculate that macrophages recruited to the repeatedly injured lung are regulated by placental growth factor (PlGF; which was upregulated in chronically injured lungs), as PlGF is a ligand for vascular endothelial growth factor receptor 1 (VEGFR1; damaged lungs contained high numbers of macrophages expressing this receptor).

Importantly, TC14012 attenuated lung fibrosis and JAG1 induction in mice with various levels of $\beta$-cateinin-induced JAG1 expression in PCECs: from mice reconstituted with Vegfr $1^{-/-}$monocytes (which had low levels of fibrosis) to those with stabilized $\beta$-cateinin (which had high levels of fibrosis). These data suggest that CXCR7 activation can override the profibrotic activity of macrophages to reduce JAG1 expression in PCECs and subsequent lung fibrosis. CXCR7 agonists could therefore be useful to treat lung fibrosis in the future.

Megan Cully

ORIGINAL ARTICLE Cao, Z. et al. Targeting of the pulmonary capillary vascular niche promotes lung alveolar repair and ameliorates fibrosis. Nat. Med. 22, 154-162 (2016)

FURTHER READING Kreuger, J. \& Phillipson, M.

Targeting vascular and leukocyte communication in angiogenesis, inflammation and fibrosis. Nat. Rev. Drug Discov. 15, 125-142 (2016) 